\title{
Elastofibroma dorsi un tumor infrecuente o una masa involuntariamente ignorada
}

Federico Lubinus B. ${ }^{*}$, Óscar Ortiz C. ${ }^{1}$, Diana Valenzuela S. ${ }^{1}$, Kevin Hurtado O. ${ }^{1}$, Aristides Sotomayor R. ${ }^{1}$, Silvia Vera C. ${ }^{2}$, Erick Villareal I. ${ }^{3}$, Laura Ramírez $\boldsymbol{P}^{4}$

1. Radiólogo. Departamento de radiología, Clínica Carlos Ardila Lülle, Bucaramanga. Colombia.

2. Médico. Universidad Industrial de Santander, Bucaramanga, Colombia.

3. Estudiante de medicina, Universidad Autónoma, Bucaramanga, Colombia.

4. Médico. Universidad Autónoma, Bucaramanga, Colombia.

\section{Elastofibroma dorsi: a rare tumor or an involuntarily unlooked mass}

\section{Resumen:}

Objetivo: Describir mediante una serie de casos la frecuencia de elastofibroma dorsi utilizando la tomografía como método diagnóstico. Métodos: Se analizó de forma retrospectiva las tomografías computarizadas (TC) de tórax con y sin contraste, realizadas en el período comprendido entre el periodo de junio de 2012 hasta febrero de 2013 en busca de hallazgos compatibles con elastofibroma dorsi. Resultados: Se evaIuaron 1085 TC, de los cuales 23 casos fueron positivos para elastofibroma dorsi con una prevalencia del $2,1 \%$. Se presentó compromiso bilateral en un $43,6 \%$ mientras que el compromiso unilateral fue del $56,4 \%$, de los cuales el $84,6 \%$ fueron derechos y 15,4\% izquierdos. Conclusiones: El elastofibroma es un tumor benigno de tejidos blandos con localización y apariencia característica en la TC, considerado en la literatura mundial como raro e infrecuente, sin embargo, nuestro estudio encontró una prevalencia significativa en la población general.

Palabras clave: Elastofibroma dorsi; Región subescapular; Tejido blando; Tumor benigno.

\section{Abstract:}

Aims: To describe through a series of cases the frequency of elastofibroma dorsi using computed tomography as a diagnostic method. Methods: The Chest computed tomography (CT) scans with and without contrast seen from June 2012 to February 2013 were retrospectively analyzed searching for Elastofibroma Dorsi. Results: 1085 CTs were evaluated, 23 cases were positive for elastofibroma dorsi with a prevalence of $2,1 \%$. Bilateral lesions were present in $43,6 \%$ while a unilateral lesion was present in $56,4 \%$. In $84,6 \%$ of the patients the ED was in the right side and in 15,4\% It was in the left. Conclusions: Elastofibroma Dorsi is a benign soft tissue tumor showing a usual location and appearance on CT. It is considered to be a rare and infrequent tumor in the world literature however, our study found a significant prevalence on the general population.

Keywords: Benign tumor; Elastofibroma dorsi; Soft tissue; Subscapular region.

Lubinus $F$, et al. Elastofibroma dorsi un tumor infrecuente o una masa involuntariamente ignorada. Rev Chil Radiol 2020; 26(4): 141-144.

*Correo electrónico: Federico Lubinus, MD / flubinus@hotmail.com.

Trabajo enviado el 18 de marzo de 2020. Aceptado para publicación el 17 de junio de 2020.

\section{Introducción}

El elastofibroma dorsi (ED) es un tumor benigno descrito por primera vez en $1962^{1}$; clasificado por la OMS como un tumor fibroblástico / miofibroblástico benigno según la clasificación de tumores de tejidos blandos publicada en $2013^{2}$. Se ha reportado en la literatura su baja frecuencia de presentación, siendo descrito la mayoría de las veces como reporte de caso o serie de $\operatorname{casos}^{3,4,5,6,7,8,9,10,11}$. Existen pocos estudios donde se hable de una prevalencia significativa en la población general ${ }^{12,13,14}$. Su localización más común es en la región subescapular entre la musculatura y 
la pared torácica, y con menor frecuencia a nivel infra olecraneana, tuberosidad isquiática, pared torácica, deltoides, entre otros ${ }^{15}$.

Los síntomas dependen del tamaño y localización de la lesión, siendo la mayoría asintomática, aunque se pueden presentar con dolor a nivel escapular o de espalda ${ }^{16,17}$, el cual puede irradiarse a hombro llevando a diagnóstico erróneo de otras patologías como sarcoma de tejidos blandos, bursitis o lesión del manguito rotador ${ }^{2}$.

El propósito de nuestro estudio es describir mediante una serie de casos la frecuencia de elastofibroma dorsi en población adulta no oncológica mediante tomografía computada de tórax.

\section{Materiales y métodos}

Se realizó una revisión retrospectiva de las TCs de tórax con y sin contraste realizadas entre el 1 de junio de 2012 y el 28 de febrero de 2013 a pacientes mayores de 18 años en busca de hallazgos compatibles con elastofibroma dorsi. Se excluyeron los pacientes con diagnóstico presuntivo de cáncer. El diagnóstico de los casos positivos fue realizado en consenso con un radiólogo experto. Todas las tomografías fueron realizadas en un equipo multicorte TOSHIBA de 256 detectores, con cortes de $10 \mathrm{~mm}$ de espesor y reconstrucciones en el plano sagital y coronal, con un Kv de 120 y 250 mAs, utili- zando ventana de 400 con nivel de 40 .

Todos los pacientes se escanearon en posición supina con los brazos en extensión sobre la cabeza y se administró contraste endovenoso, utilizando una infusión de 3,5 cc/seg para un total de $70 \mathrm{cc}$.

Entre los criterios diagnósticos se incluyó la presencia de masa con densidad similar a las estructuras musculares adyacentes, sin variaciones con la administración del medio de contraste, de márgenes convexos y relación profunda con el borde inferior de la escapula.

Los datos fueron analizados buscando la presencia de las lesiones, así como su lateralidad y la variabilidad según el sexo de los casos reportados.

\section{Resultados}

Se evaluaron 1085 TC de pacientes entre los 18 y 99 años (47\% hombres y $53 \%$ mujeres). Se encontraron 23 pacientes positivos para el diagnóstico de elastofibroma dorsi, lo cual corresponden al 2,1\% con una relación mujer/hombre de 1,4:1. La lesión se presentó de manera unilateral en el $56,4 \%$, de estas, el $15,4 \%$ fueron izquierdas y el $84,6 \%$ derechas. Se hallaron en forma bilateral 10 casos correspondiendo al 43,6\% (Figura 1).

El diámetro longitudinal promedio de las lesiones fue de $3,3 \mathrm{~cm}$ (rango 1,2 a 6,4 cm), todas las densidades fueron homogéneas con respecto al músculo sin evidenciarse variación con la administración de medio de contraste (Figura 2 y 3 ).

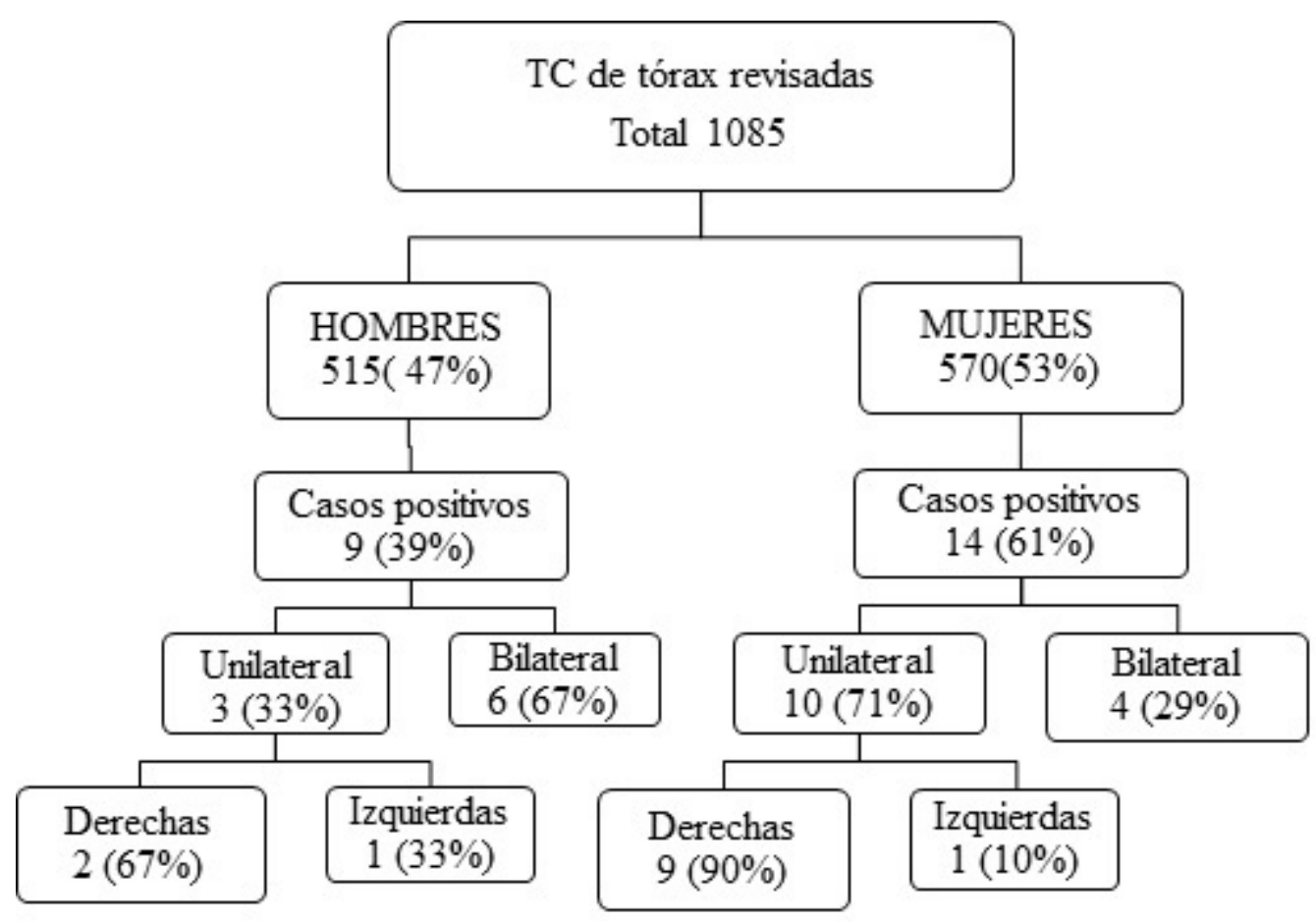

Figura 1: Porcentaje por sexo y localización del elastofibroma dorsi. 


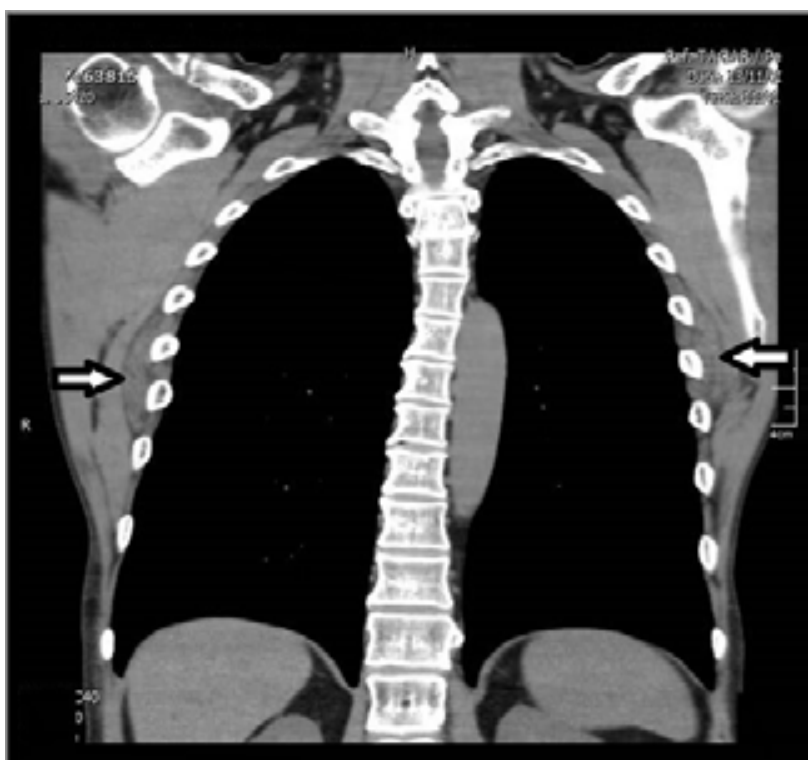

Figura 2: Corte coronal en tomografía donde se aprecia masa densidad de tejidos blandos definida inferior a planos musculares profundos con criterios de elastofibroma dorsi bilateral (Flecha blanca).

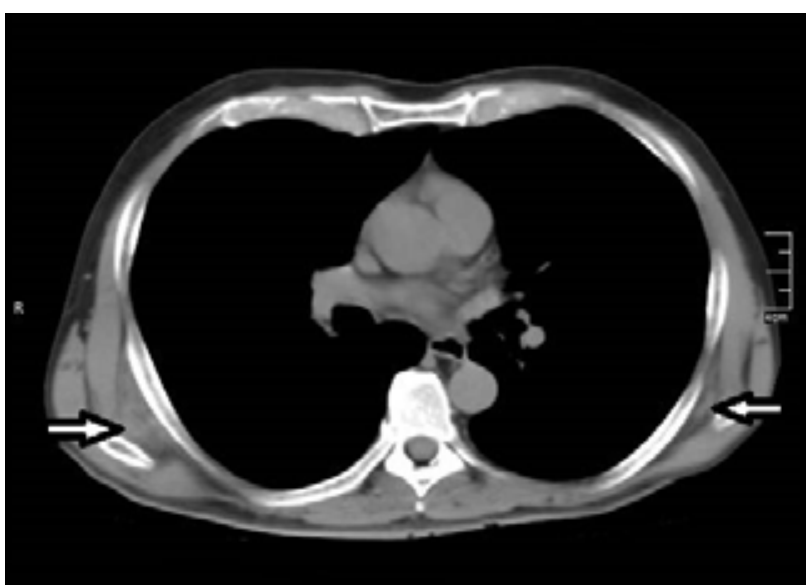

Figura 3: Corte transversal en tomografía donde se aprecia a nivel planos musculares profundos un elastofibroma dorsi bilateral (Flecha blanca).

La localización en hombres fue bilateral en un $67 \%$ ( 6 casos). En mujeres $71 \%$ fueron unilaterales, y de estos, el $90 \%$ (9 casos) fueron del lado derecho.

\section{Discusión}

El elastofriboma dorsi es un tumor benigno de predominio en el sexo femenino, en la literatura se ha reportado una relación mujer/ hombre de hasta 13:13,17. Sin embargo, en nuestro estudio se encontró una distribución similar, con una relación 1,4: 1 .

La prevalencia de esta patología es muy variable, se ha encontrado en más del $2 \%$ de las tomografías de pacientes mayores de 60 años $^{13}$, sin embargo, los estudios de autopsias han encontrado una prevalencia mayor que va del $13 \%$ al $17 \%$ de los casos, con presencia de cambios morfológicos pre-elastofibroma en hasta el $81 \%$ de las autopsias ${ }^{14,15}$, lo que sugiere un importante sub diagnóstico.

En la revisión de los exámenes tomográficos de tórax se incluyeron pacientes mayores de 18 años, sin embargo, el caso de presentación más temprana en nuestra serie fue de 48 años. La mayoría de los reportes médicos descritos en la literatura describen una edad promedio alrededor de los 50 años que para nuestro estudio fue únicamente 3 casos (13\%), lo cual supone que los casos reportados en la literatura corresponden principalmente a pacientes sintomáticos a quienes se les realizaron estudios diagnósticos ya sea por la presencia de dolor interescapular y/o por presentar una masa palpable mientras que nuestro estudio corresponde a una revisión retrospectiva de TC de tórax en pacientes asintomáticos que consultaron por otras causas no relacionadas.

Nuestros hallazgos son similares a los encontrados por Brandser et al..$^{13}$ ya que en su reporte de casos la frecuencia encontrada fue del $2 \%$. Otra de las características que es constante y no presenta variaciones en los diferentes estudios realizados es el tamaño de las lesiones, las cuales en promedio para nuestro estudio son de $3,3 \mathrm{~cm}$ en su eje mayor y siguen siendo similares en las series y reportes de casos, donde al parecer el tamaño de la lesión no influiría en el diagnóstico. Una limitante de nuestro estudio es que no fue posible correlacionar el tamaño de la lesión con la existencia o no de sintomatología.

La patogénesis del ED aún no es muy clara, sin embargo, el microtrauma repetitivo en la articulación escapulo torácica puede causar hiperproliferación reactiva de tejido fibroelástico y ser el origen de esta patología ${ }^{18}$, teoría que es aceptada y tiene correlación con los hallazgos de mayor incidencia en el lado derecho, teniendo en cuenta que la mayoría de la población es dominante derecha estando así expuesta a mayor microtrauma en este lado durante toda su vida. Sin embargo, en nuestro trabajo los casos encontrados en hombres fueron bilaterales en su mayoría, mientras que en mujeres fueron unilaterales de predominio derecho, lo que hace pensar que esta teoría no es la única explicación a su patogenia $y$ teniendo en cuenta el aumento de frecuencia de aparición con la edad y el hecho de tener una mayor frecuencia de aparición en los estudios de autopsia supone un factor degenerativo o compresivo crónico como desencadenante de la lesión desmoplasica causante de la patología. Adicionalmente se proponen otras hipótesis como la insuficiencia vascular y la predisposición familiar que también pueden jugar un papel importante en su etiología ${ }^{19}$. 
El hallazgo de esta lesión en la TC es generalmente incidental, al identificar una masa solida con márgenes no bien definidos, indistinguible del plano costal profundo y separado de los tejidos blandos superficiales por una capa de grasa, sin evidencia de infiltración o edema perilesional reactivo en sus márgenes. La presencia de patrón fasciculado del ED en TC y resonancia magnética permite diferenciarlo de otros tumores de tejidos blandos como el lipoma, tumor desmoide, sarcoma de tejidos blandos y metástasis ${ }^{18}$. En nuestro estudio no se encontraron lesiones tumorales peri escapulares con características distintas a ED, lo que puede ser explicado por la exclusión de pacientes de centro de cáncer o con hallazgo del mismo en la revisión del examen.

El tratamiento depende de la sintomatología, debido a que no se ha descrito transformación maligna, los pacientes asintomáticos no requieren tratamiento ${ }^{20}$, mientras que los pacientes sintomáticos o en quienes la malignidad no puede ser excluida requieren manejo quirúrgico ${ }^{21}$.

En estudios recientes utilizando la tomografía por emisión de positrones los ED han mostrado una captación difusa de Fluorodeoxiglucosa (FDG) de bajo a moderado grado, sin embargo, también se puede observar una alta captación de FDG la cual puede ser causada por una alta vascularidad y una proliferación reactiva fibroblástica anormal dentro de la masa ${ }^{22}$.

Una de las limitaciones de nuestro estudio es que no se dispuso de biopsia para la confirmación histológica del diagnóstico de elastofibroma dorsi, sin embargo, se considera que las características tomográficas descritas son suficientes para un diagnóstico de certeza. Otra limitación es que no se obtuvieron datos clínicos para determinar cuál es la frecuencia de lesiones sintomáticas.

\section{Conclusión}

El ED es un tumor de tejidos blandos poco reportado en la literatura, encuentrándose con mayor frecuencia como reporte de caso o serie de casos, probablemente por ser una patología asintomática y de curso benigno, en la que es requerido el estudio y manejo sólo si hay síntomas.

En nuestro estudio se encontró una prevalencia significativa de esta enfermedad, lo que indica que el ED no es un tumor infrecuente sino una lesión involuntariamente ignorada.

\section{Referencias}

1. Jarvi $\mathrm{OH}$, Saxen AE. Elastobibroma dorsi. Acta Pathol Microbiol Scand. 1961; 144(Suppl 5): 83-84.

2. Fletcher CDM, Bridge JA, Hogendoorn P, Mertens F. WHO classification of tumours of soft tissue and bone. 4th edn. Lyon: IARC. 2013: 110-111.

3. Cavallascaa JA, Sohnb DI, Borgiaa AR, Maliandia M,
Musuruanaa JL. Elastofibroma dorsi: Revisión de 4 casos. Reumatol Clin. 2012; 8(6): 358-360.

4. Muratori F, Esposito M, Rosa F, Liuzza F, Magarelli N, Rossi B, et al. Elastofibroma dorsi: 8 case reports and a literature review. J Orthopaed Traumatol. 2008; 9: 33-37.

5. Guha AR, Raja RCS. Elastofibroma dorsi: A case report and review of literature. Int J Clin Pract. 2004; 58: 218-220.

6. Hayes AJ, Alexander N, Clark MA, Thomas JM. Elastofibroma: A rare soft tissue tumour with a pathognomonic anatomical location and clinical symptom. Eur J Surg Oncol 2004; 30: 450-453.

7. Heck S, Thomas G, Mader K, et al. Bilateral elastofibroma as unusual cause of shoulder pain. Plast Reconstr Surg. 2003; 112: 1959-1961.

8. Oueslati S, Douira-Khomsi W, Bouaziz MC, Zaouia K. Elastofibroma dorsi: a report on 6 cases. Acta Orthop Belg. 2006; 72(2): 237-242.

9. Turna A, Yilmaz M.A, Urer N, et al. Bilateral elastofibroma dorsi. Ann Thorac Surg. 2002; 73: 630-632.

10. Vastamaki M. Elastofibroma Scapulae. Clin Orthop Relat Res. 2001; 392: 404-408.

11. Malghem J, Baudrez V, Lecouvet F, et al. Imaging study findings in elastofibroma dorsi. Joint Bone Spine. 2004; 71: 536-541.

12. Tepe M, Polat M, Calisir C, Ulukan Inan, Bayav M. Prevalence of elastofibroma dorsi on CT: Is it really an uncommon entity? Acta orthop traumatol turc. 2019; 53(3): 195-198.

13. Brandser EA, Goree JC, El-Khoury GY. Elastofibroma dorsi: prevalence in an elderly patient population as revealed by CT. Am J Roentgenol 1998; 171(4): 977-980.

14. Giebel GD, Bierhoff E, Vogel J. Elastofibroma and preelastofibroma-a biopsy and autopsy study. Eur J Surg Oncol 1996; 22(1): 93-96.

15. Jarvi $\mathrm{OH}$, Lansimies $\mathrm{PH}$. Subclinical elastofibromas in the scapular region in an autopsy series. Acta Pathol Microbiol Scand. 1975; 83(1): 87-108.

16. Cano A, Bravo F, Garrido J, Ortega R. Elastofibroma dorsal: hallazgos en TC y RM. A propósito de dos casos. Radiología. 2001; 43(7): 353-355.

17. Ramos R, Ureña A, Macía I, Rivas F, Ríus X, Armengol J. Fibroelastoma dorsi: un tumor infrecuente e infradiagnosticado. Arch Bronconeumol. 2011; 47(5): 262-263.

18. Battaglia M, Vanel D, Pollastri P, Balladelli A, Alberghini M, Staals EL, et al. Imaging patterns in elastofibroma dorsi. Eur J Radiol. 2009; 72: 16-21.

19. Daigeler A, Vogt PM, Busch K, Pennekamp W, Weyhe D, Lehnhardt M, et al. Elastofibroma dorsi-differential diagnosis in chest wall tumours. World J Surg Oncol. 2007; 5:15.

20 Koksel O, Demir Apaydin F, Ayan E, Demir M, Ozdulger A. Elastofibroma dorsi: Review of eight cases. Surg Today. 2010; 40(5): 423-427.

21. Mortman KD, Hochheiser GM, Giblin EM, Manon-Matos $Y$, Frankel KM. Elastofibroma dorsi: clinicopathologic review of 6 cases. Ann Thorac Surg. 2007; 83(5): 1894-1897.

22. Tripathy S, Parida GK, Naswa N, Subudhi K, Sreedharan AR, Reddy S. Elastofibroma Dorsi: Findings on 18FFluorodeoxyglucose Positron Emission TomographyComputed Tomography. Indian J Nucl Med. 2019; 34(3): 258-259. 